\title{
A Third Labyrinthine Window: An Overview of Perilymph and Labyrinthine Fistulae and Superior Semicircular Canal Dehiscence
}

\author{
Bachi T Hathiram, Vicky S Khattar
}

\begin{abstract}
The membranous labyrinth is contained within the bony labyrinth and surrounded by perilymph. The only two 'potentially yielding' parts of the otherwise solid bony labyrinth are the oval and round windows, which by their relative movements, pressure differentials and resilience are responsible for all the functions attributed to the inner ear. In pathologies, such as trauma, infection or occasionally congenital dehiscence, there may develop a 'third window' that may serve as an abnormal communication for the inner ear fluids and manifest with audiovestibular symptoms. Three such distinct entities have been identified, namely 'superior semicircular canal dehiscence syndrome, perilymphatic fistulae and labyrinthine fistulae'. This overview intends to discuss these above-mentioned entities, as regards their characteristic presentations and principles of management.
\end{abstract}

Keywords: Perilymphatic fistula, Labyrinthine fistula, Superior semicircular canal dehiscence.

How to cite this article: Hathiram BT, Khattar VS. A Third Labyrinthine Window: An Overview of Perilymph and Labyrinthine Fistulae and Superior Semicircular Canal Dehiscence. Otorhinolaryngol Clin Int J 2012;4(2):100-105.

\section{Source of support Nil}

Conflict of interest: None declared

\section{INTRODUCTION}

The inner ear is a complex, fluid-filled structure surrounded by a bony labyrinth and located within the temporal bone. The cochlea is the acoustic end organ, and the vestibular end organs consist of the three semicircular canals with their ampullae, the saccule and the utricle. The endolymphatic sac, also part of the inner ear, is thought to be involved in the fluid regulation of the inner ear. A ny disorder of the vestibular end organs is perceived as vertigo.

Inner ear disorders are common and patients with vestibular failure often present to a neurology clinic because of their dizziness, unsteadiness and/or oscillopsia. V estibular disorders can be divided into peripheral and central vestibular disorders. Most of the peripheral vestibular disorders have a clinical diagnosis, and a thorough history and examination will often help in arriving at an accurate diagnosis. Correct diagnosis allows for early treatment of the peripheral and central vestibular disorders. A s inner ear damage is generally irreversible, early diagnosis allowing prompt treatment is important.
Vertigo is not a separate disease process, but a multisensory and sensorimotor syndrome with various etiologies and pathogeneses. ${ }^{1}$ The term 'dizziness' refers either to an unpleasant disturbance of spatial orientation or to the erroneous perception of movement, which is more specifically called 'vertigo'. V ertigo involves a perceived movement either of one's own body, such as swaying or rotation, or of the environment, or both. A longside headache, dizziness and vertigo are among the more common symptoms with which patients present to physician in general, not just to neurologists. Their lifetime prevalence is approximately 20 to $30 \%{ }^{2}$

Recurrent episodes of vertigo and oscillopsia, induced by stimuli that produce changes in intracranial or middle ear pressure (e.g. coughing or loud noises), are associated with a defect in the labyrinthine canal causing a third mobile window in the labyrinth. This appears with superior canal dehiscence syndrome and perilymphatic fistula. ${ }^{3}$ Superior canal dehiscence syndrome has been recognized relatively recently, and these patients often have a hypersensitivity to bone-conducted sounds and have a mild low-frequency hearing loss. Perilymph fistula is often a manifestation of chronic otitis media, cholesteatoma or temporal bone fractures. However, idiopathic cases have also been reported.

Middle ear pathology, such as unsafe chronic suppurative otitis media (CSOM) is a common cause of audiovestibular dysfunction and, hence, it is mandatory to take a thorough history so as to accurately diagnose this condition. Cholesteatoma usually causes a labyrinthine fistula by erosion of the bony labyrinth. It is commonly seen to affect the bone of the lateral semicircular canal. The treatment of this pathology is essentially surgical and early surgery with closure of the labyrinthine fistula would help in preventing the loss from worsening.

Perilymph fistula is often a manifestation of barotrauma or temporal bone fractures. However, idiopathic cases have also been reported. The pathophysiological mechanisms are thought to be increased elasticity of the otic capsule or leakage of perilymph, usually at the oval or round window. ${ }^{4}$ The fistula test (Hennebert's sign) that involves positive and negative pressure in the external ear canal, causing eye movements and/or vertigo, supports the diagnosis. With 
A Third Labyrinthine Window: An O verview of Perilymph and Labyrinthine Fistulae and Superior Semicircular Canal Dehiscence

positive pressure, a conjugate deviation of the eyes toward the opposite ear is followed by a corrective fast eye movement. A ccordingly, the direction of the nystagmus is toward the affected ear and can be horizontal, torsional or vertical, depending on the location of the fistula. There are at present no available tests with high specificity to diagnose perilymphatic fistula. If symptoms are disabling, surgical exploration of the ear to detect the fistula and seal it may be considered. However, even at the time of surgical exploration, a perilymphatic fistula is often difficult to identify.

In this article, we have attempted to review these causes of vertigo, their diagnosis and modalities of management, since inner ear damage is known to be irreversible and hence, an early diagnosis will help to prevent further worsening of the audiovestibular loss.

\section{Superior Semicircular Canal Dehiscence Syndrome}

Superior semicircular canal dehiscence syndrome is caused by a defect of bone overlying the superior (anterior) semicircular canal, enabling changes in intracranial pressure to be pathologically transduced to the superior semicircular canal (Figs 1 and 2). Superior semicircular canal dehiscence $(S C D)$ syndrome is a recently recognized entity where patients often present with vestibular symptoms typically provoked by sound and/or static pressure, ${ }^{5,6}$ Loud sounds applied to the symptomatic ear result in torsional nystagmus appropriate for stimulation of the superior semicircular canal in most cases. SCD is thought to be congenital because it is often bilateral and is also seen in asymptomatic people.

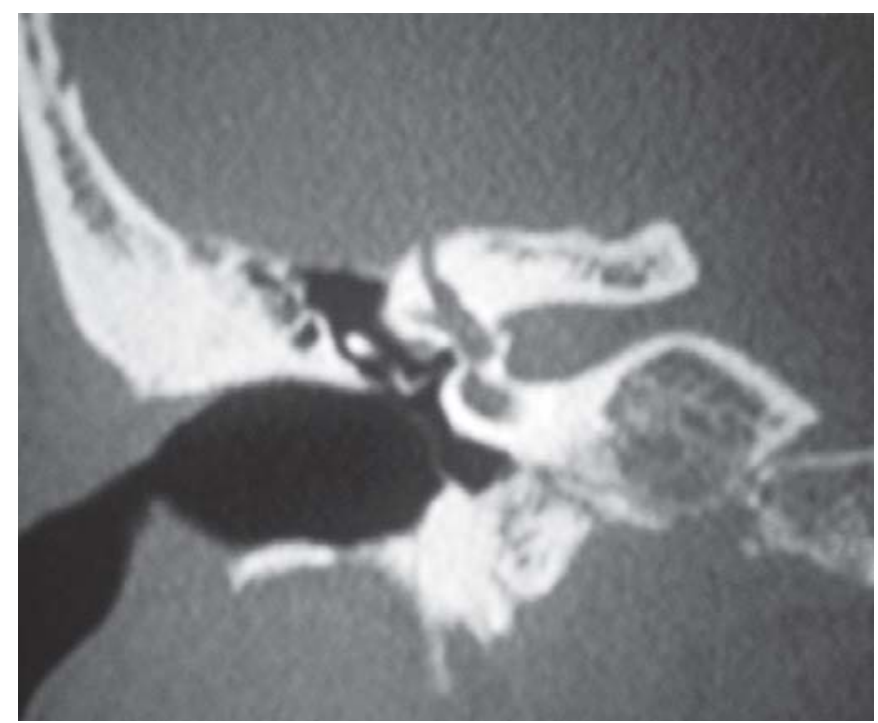

Fig. 1: Coronal view of a high resolution CT scan of the temporal bone showing a dehiscence in the roof of the right superior semicircular canal
The syndrome of dehiscence of the superior semicircular canal is a clinical condition encompassing a variety of vestibular and auditory symptoms, including an air-bone gap at low frequencies. It has been hypothesized that the dehiscence acts as a 'third window' into the inner ear that shunts acoustic energy away from the cochlea at low frequencies, causing hearing loss. It is somewhat puzzling that an air-bone gap on audiometry can be produced by a pathologic condition affecting the inner ear rather than the middle ear. Definitive proof that the air-bone gap in these patients is indeed the result of the SCD is the closure of the gap when the dehiscence is repaired. ${ }^{7,9}$

The Weber's tuning fork test typically shows lateralization to the affected ear. Other unusual symptoms are of hearing their own eye movements or their pulse. The diagnosis is made using high resolution temporal bone computed tomographic scan, which shows the defect of the bone overlying the superior/anterior semicircular canal.

In a study conducted at the M assachusetts Eye and $E$ ar Infirmary USA (2007), it was found that sound induced umbo velocity $(\mathrm{Vu})$ measurements using laser-Doppler vibrometry that can be helpful for the diagnosis of SCD in patients who are presented with an unexplained air-bone gap, an intact tympanic membrane, and an aerated middle ear. In particular, it was found to be useful for differentiating otosclerosis from SCD. It is known that the clinical presentation of SCD can mimic that of otosclerosis; and SCD patients have, in some instances, undergone stapedectomy without improvement in their hearing. ${ }^{7-9}$ In otosclerosis, the $V$ u magnitude is decreased compared with the mean normal (15) because of an increase in inner-ear input impedance by fixation of the stapes footplate, whereas

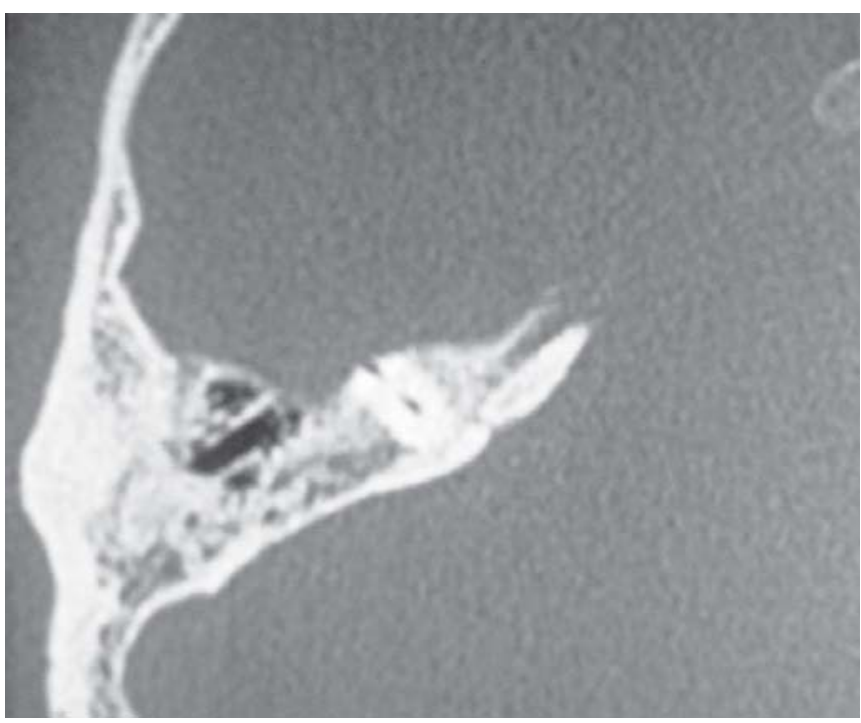

Fig. 2: Axial view of the same patient (above) showing the defect in the anterior crus of the right superior semicircular canal 
in $S C D$, the $V$ u magnitude is increased because of a decrease in inner-ear input impedance. Therefore, $V$ u measurements can be helpful in distinguishing between these two clinical conditions. However, it is important to remember that although $V u$ measurements can help discriminate whether an air-bone gap results from stapes fixation or $S C D$, it al one cannot be used to diagnose SCD. This is because the variation in $\mathrm{Vu}$ measurements among normal subjects is larger than the $4 \mathrm{~dB}$ increase in $\mathrm{Vu}$ (compared with the mean normal) observed in patients with SCD. Thus, one cannot reliably distinguish between $V u$ in normal vs SCD ears in the absence of any additional diagnostic tests, such as an audiogram, acoustic reflex testing, vestibular-evoked myogenic potentials or high-resolution computed tomographic scans. However, in the presence of an air-bone gap, $\mathrm{V} u$ measurement is an easy and effective way of differentiating between SCD and otosclerosis. ${ }^{10}$

Conservative management involved the use of diuretics, low salt diet, and vestibular suppressants, in addition to avoiding the offending stimulus. Surgical management of SCD by either plugging the defect with bone wax/paste or resurfacing with a bone graft has been shown to be successful in around $50 \%$ of cases. ${ }^{11,12}$ The traditional middle cranial fossa approach to surgically treat this condition is now gradually being replaced by the transmastoid approach which is less morbid for the patient and gives equally good results in experienced hands.

\section{Perilymph Fistula}

Even though diagnostic and surgical techniques have improved rapidly, perilymphatic fistula (PLF) remains one of the most challenging problems in otological practice. A PLF is an abnormal communication between the inner ear fluids and the middle ear space. ${ }^{13}$ The first report of otorrhea with cerebrospinal fluid was by Escat in 1897, while in 1933 $\mathrm{K}$ line reported another case of a 54 -year-old mal e with clear fluid otorrhea. ${ }^{14,15}$ L ewis, in 1961, and Sheehy and House, in 1962, reported perilymph fistulas after stapedectomy, Fee, in 1968, after trauma, and Pullen, in 1979, after barotrauma in divers. ${ }^{16-19}$ In 1970 Stroud and Calcaterra reported spontaneous leakage and in 1971, Goodhill stressed the point of a relationship between sudden hearing loss and round window rupture. ${ }^{20,21}$ Controversy prevails regarding the accuracy of the clinical diagnosis of PLF and many studies have attempted to achieve a better diagnosis of this entity. ${ }^{22-25}$

The diagnosis of PLF is based on a careful subjective evaluation of vestibular function tests, and the intraoperative visualization of the flow of perilymph from the oval and/or round windows at the time of an exploratory tympanotomy. However, there is no consensus regarding the indications of an exploratory tympanotomy in literature even today.
PLF commonly occurs following trauma or some physical effort, such as straining, coughing, sneezing, etc. The patient usually complains of a popping sensation in the ear followed by a blocking or decrease in hearing associated with vertigo and/or tinnitus. Nystagmus may be seen on examination. In the literature vertigo and nystagmus are regarded as an indicator of PLF ${ }^{13,26-28}$ reported that only $18 \%$ of patients could be operated on the basis of this complaint alone. A pure tone audiometric analysis would reveal a sensorineural hearing loss in majority of patients. The EN G fistula test was reported to be diagnostic in $90.8 \%$ of cases $^{29}$ but later reports lowered the exactness of this test from 26 to $50 \% .{ }^{30}$ However, in a study conducted, ${ }^{31}$ a positive fistula test in ENG was suggestive of a PLF in $75 \%$, with the presence of apositive caloric test in $78 \%$ and with the additional presence of positional nystagmus in $100 \%{ }^{32}$ suggested electrocochleography as a test for PLF. A positive finding was found in $82 \%$, but there were $30 \%$ false negative findings. However, clinical examination plays an important role in the diagnosis of this condition since almost all patients will have a positive fistula test along with a positional nystagmus. Occasionally, a PLF may also occur secondary to CSOM (Fig. 3).

The common sites of occurance of a PLF are the round window, the oval window, both the windows or the lateral semicircular canal. The confirmative diagnosis of a PLF is arrived at only intraoperatively when, on an exploratory tympanotomy, there is an intraoperative visualization of clear fluid from the round or oval window in the Trendelenburg position or after the patient was instructed to perform a $V$ alsalva maneuver at the time of the surgery, in order to visualize the fistula in the round window membrane. Also, the absence of a round window reflex is indicative of a PLF. If a patient presents with a sensorineural

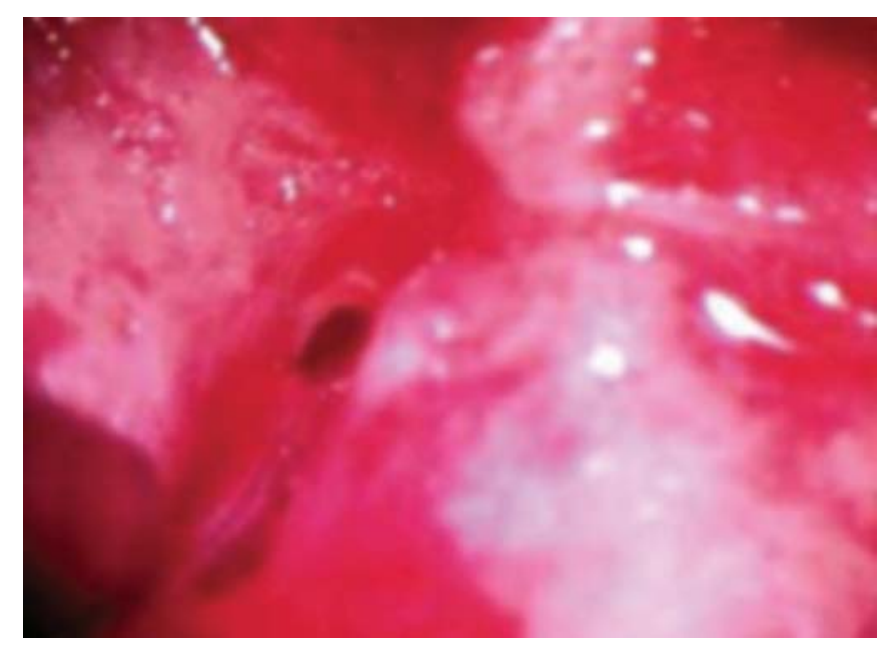

Fig. 3: An intraoperative microscopic view showing an erosion in the anterior part of the footplate of the stapes in a case of chronic suppurative otitis media with extensive granulations. The fistula was revealed after clearance of the granulation tissue from the middle ear 


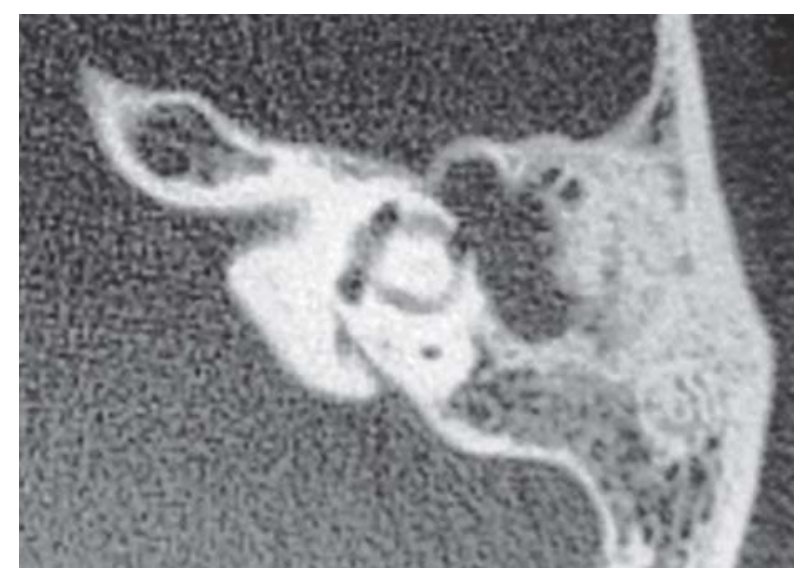

Fig. 4: An axial view of the HRCT scan of the temporal bone showing a fistula in the left horizontal semicircular canal in a case of CSOM

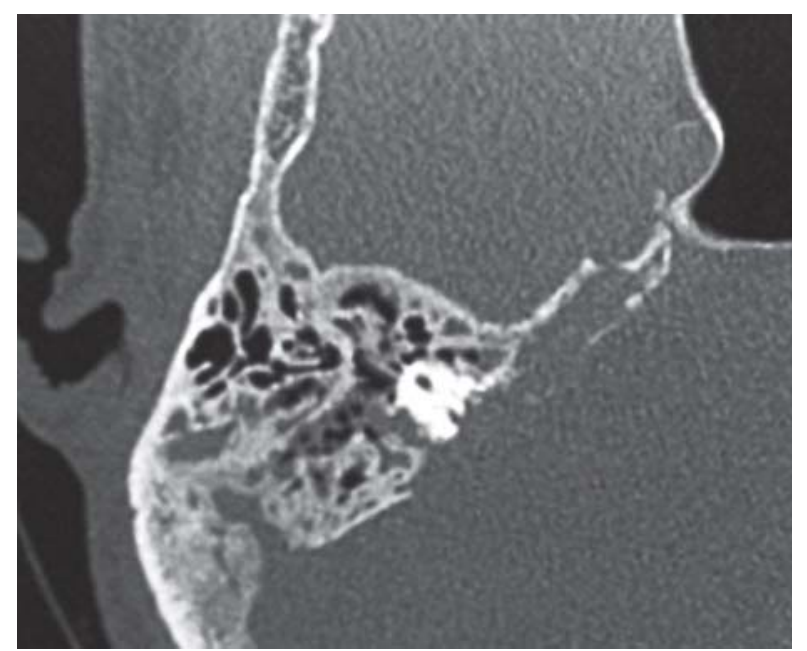

Fig. 5: Axial view of the HRCT temporal bone scan of a patient with petrositis with erosion of the superior semicircular canal. The patient presented similar to one suffering from superior semicircular canal dehiscence syndrome

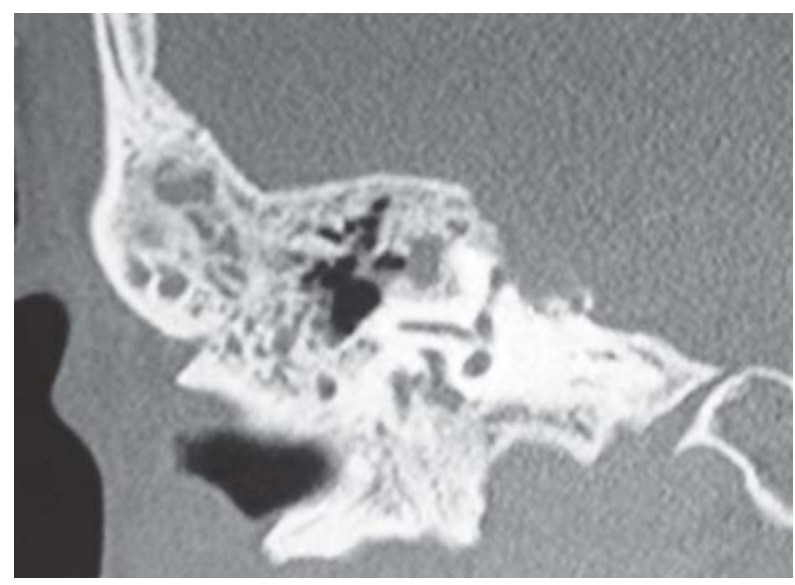

Fig. 6: The coronal view of the same patient (Fig. 5)

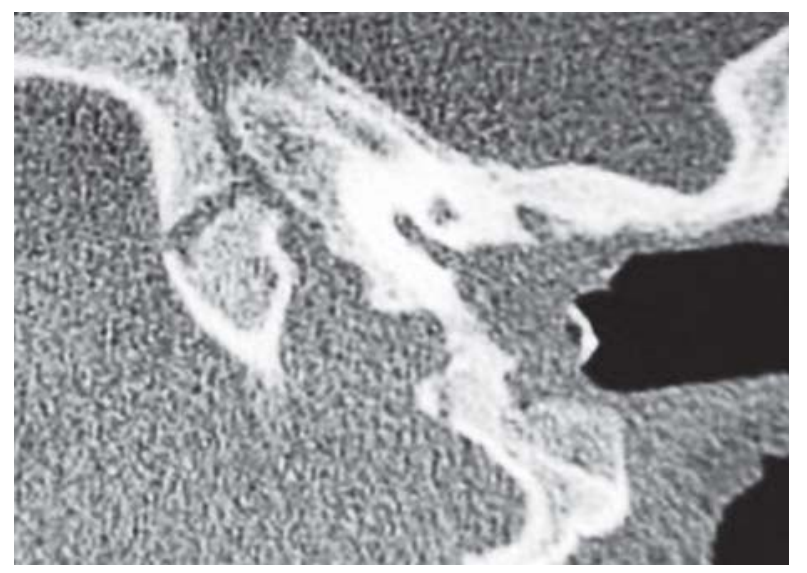

Fig. 7: An axial view of the HRCT temporal bone showing a large promontorial fistula in a case of congenital cholesteatoma

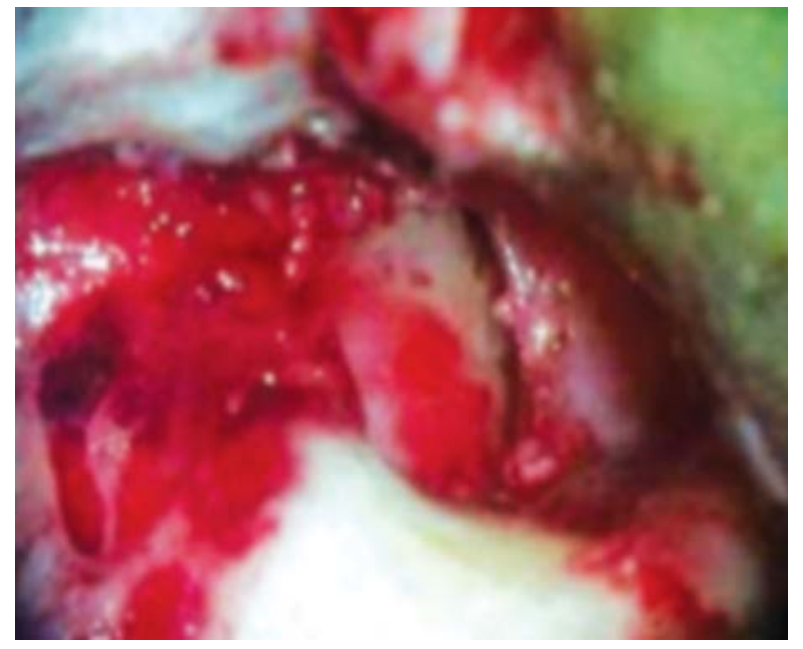

Fig. 8: Intraoperative microscopic view showing a large fistula involving the dome of the lateral semicircular canal with the endosteum intact, in a patient with unsafe CSOM with cholesteatoma

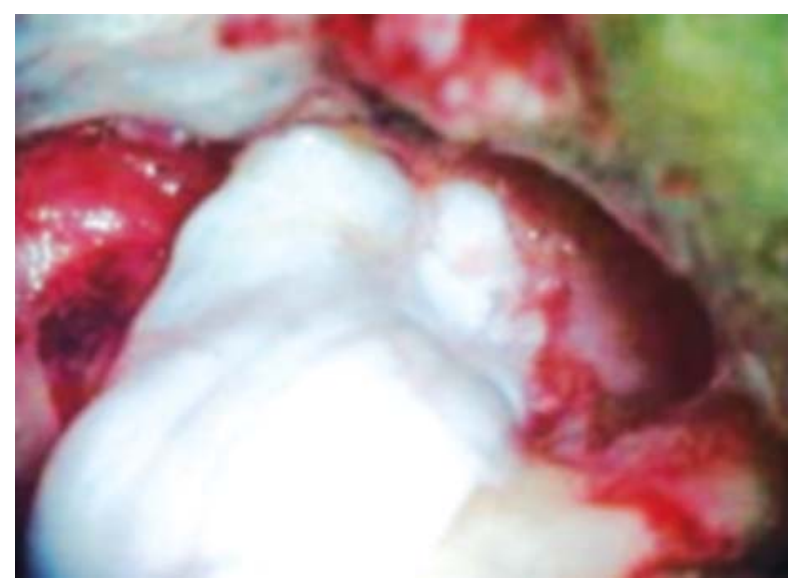

Fig. 9: After completing the mastoidectomy (Fig. 8), a temporalis fascia graft is placed to cover the middle ear space, leaving the fistula exposed 


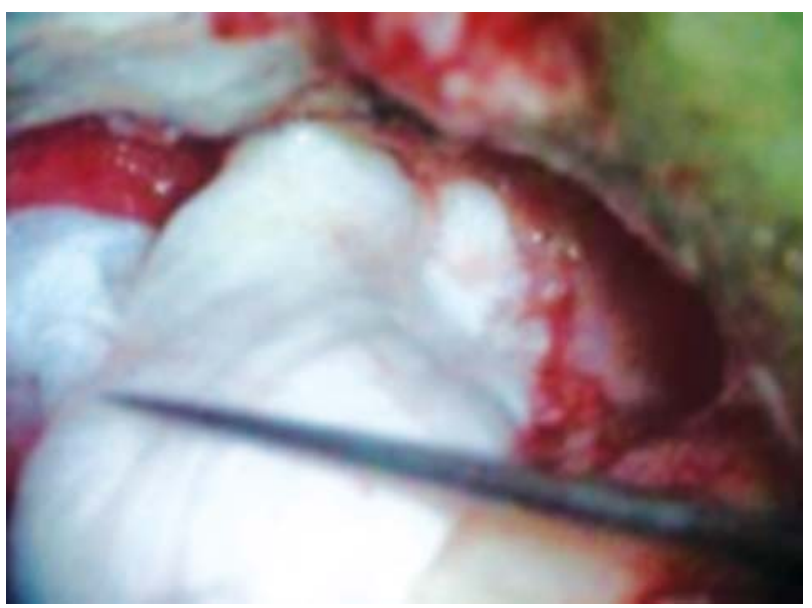

Fig. 10: A second temporalis fascia is placed to cover the fistula

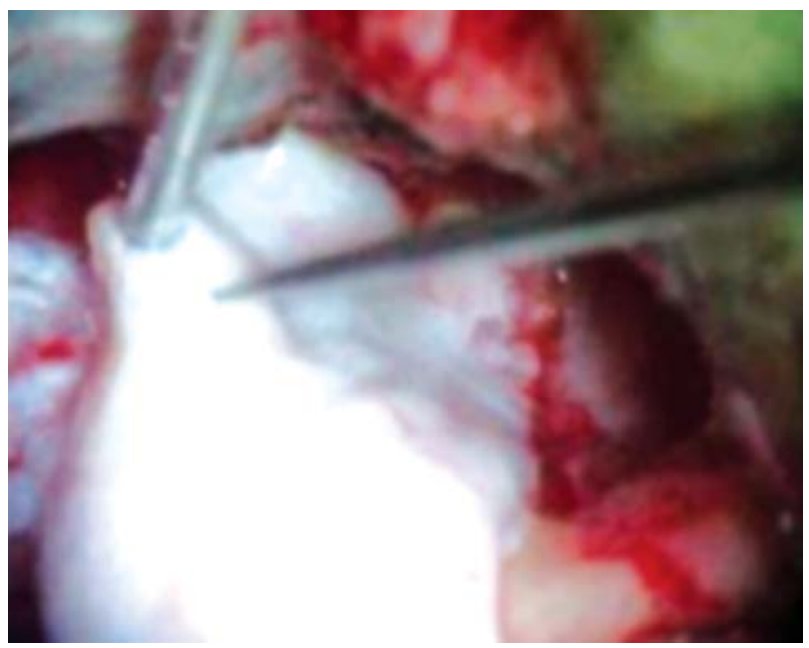

Fig. 11: The first graft is gently lifted to cover the second graft

hearing loss, vertigo and nystagmus with a positive fistula test and intact tympanic membrane following trauma, it is advisable to explore the ear for a PLF at the earliest as this condition is reversible in a number of cases when the fistula is sealed early. If a fistula of the windows is not demonstrable the lateral semicircular canal should be explored. If, on the other hand, a fistula in the windows is found and repaired, and the patient does not improve within 6 weeks, exploration of the lateral semicircular canal is recommended. The fistula is sealed with connective tissue, fat or fascia.

\section{Labyrinthine Fistula}

Middle ear pathology [e.g. otitis media with middle ear effusion (OME) and CSOM] is a common cause of audiovestibular dysfunction. The presence of an auditory abnormality indicates a peripheral rather than central cause for vestibular symptoms. It is therefore extremely important to consider the significance of prevalence or history of middle ear disease and its treatment by ear surgery in patients presenting with vestibular symptoms. Unsafe CSOM (cholesteatoma, granulations, etc.) can result in erosion of the bone and result in a labyrinthine fistula. The chronic inflammation associated with accumulation of keratin and release of enzymes from the cholesteatoma matrix causes progressive destruction, and may erode the ossicular chain and subjacent bone with consequent hearing loss, vestibular dysfunction and facial paralysis. The lateral semicircular canal is the most common site of a labyrinthine fistula occurring due to an infective/inflammatory pathology (Fig. 4). It may, however, al so be seen in other parts of the labyrinth, such as the superior (Figs 5 and 6) or posterior semicircular canals or even occasionally the promontory (Fig. 7). The patient may present with vertigo of recent onset but, there is always an associated history of otorrhea and progressively worsening hearing loss. There may be other complications of unsafe CSOM also present, such as facial palsy, etc. The pure tone audiometric evaluation will reveal a mixed hearing loss. The fistula sign may be positive in some but, may be a false negative in most patients and nystagmus may or may not be elicited. However, if a labyrinthine fistula is suspected in an unsafe CSOM, the high resolution computed tomogram (HRCT) of the temporal bone is the confirmatory diagnostic investigation of choice. The treatment is essentially surgical to prevent further worsening of the hearing. The fistula is seal ed using either bone pate, connective tissue, fascia (as a single layer or as a double layer (Figs 8 to 11 ).

\section{ACKNOWLEDGMENT}

The authors are thankful to the Dean, TN M edical College and BY L N air Charitable. Hospital for granting permission to publish this article.

\section{REFERENCES}

1. Strupp M, Brandt T. Diagnosis and treatment of vertigo and dizziness. D tsch A rzteblInt 2008;105(10):173-80.

2. Neuhauser HK. Epidemiology of vertigo. Curr Opin Neurol 2007;20:40-46.

3. Baloh RW. Superior semicircular canal dehiscence syndrome. N eurology 2004;62:684-85.

4. M inor L B . Labyrinthine fistulae: Pathobiology and management. Curr Opin Otolaryngol Head Neck Surg 2003;11:340-46.

5. M inor LB, Solomon D, Zinreich JS, et al. Sound- and/or pressure-induced vertigo due to bone dehiscence of the superior semicircular canal. A rch Otolaryngol Head Neck Surg 1998;124:249-58.

6. M inor LB. Superior canal dehiscence syndrome. A m J Otol 2000;21:9-19.

7. M inor LB, Carey JP, Cremer PD, et al. Dehiscence of bone overlying the superior canal as a cause of apparent conductive hearing loss. Otol Neurotol 2003;24:270-78. 
8. Halmagyi GM, AW ST, MCGarvie LA, et al. Superior semicircular canal dehiscence simulating otosclerosis. J Laryngol Otol 2003;117:553-57.

9. Mikulec AA, McK enna MJ, Ramsey MJ, et al. Superior semicircular canal dehiscence presenting as conductive hearing loss without vertigo. Otol Neurotol 2004;25:121-29.

10. Chien W, Ravicz ME, Rosowski JJ, Merchant SN. $M$ easurements of human middle- and inner-ear mechanics with dehiscence of the superior semicircular canal. Otol Neurotol 2007;28(2):250-57.

11. M artin JE, Neal CJ, M onacci WT, et al. Superior semicircular canal dehiscence: A new indication for middle fossa craniotomy. Case report. J N eurosurg 2004;100:125-27.

12. M ikulec AA, Poe DS, M CK anna M J . Operative management of superior semicircular canal dehiscence. Laryngoscope 2005;115:501-07.

13. M attox DE. Perilymph fistulas. In: Cumings W C, Fredrickson J M , Harker LA, K rause CJ, Schuller DE, Eds. O tolaryngologyHead Neck Surgery. CV M osby Co: St Louis, MO, USA 1986;3113-18.

14. Escat E. Ecoulementspontane de liquidecephalorachidien par le conduit auditifexterne: fistulecongenitale probable. A rchives L aryngoiogie 1897; 10:653-59.

15. Kline OR. Spontaneous cerebrospinal otorrhea. Archives Otolaryngol 1933;18:34-39.

16. Lewis $M L$ J r. Inner ear complications of stapes surgery. L aryngoscope 1961;71:377-84.

17. Sheehy JL, House HP. Causes of failure in stapes surgery. Laryngoscope 1962;72:10-31.

18. Fee GA. Traumatic perilymph fistula. A rchives Otolaryngol 1968;88:477-80.

19. Pullen FW. Sudden hearing loss in divers. Laryngoscope 1979;89:1373-77.

20. Stroud MH, Calcaterra TC. Spontaneous perilymph fistulas. Laryngoscope 1970;80:479-87.

21. Goodhill V. Sudden deafness and round window rupture. L aryngoscope 1971;81:1462-74.

22. F razer $J G$, Flood L M. A n audiometric test for perilymph fistula. J Laryngol Otol 1982;96:513-20.

23. Schweitzer VG, W oodson BT, M anhinney TD. F ree amino acid analysis of guinea pig perilymph: A possible clinical assay for the PLF enigma? Otolaryngol Head Neck Surg 1990;103: 981-85.
24. House WJ, M orris M S, Kramer JS, Shesky GL, Coggan B B, Putter JS. Perilymphatic fistula: Surgical experience in the U nited States. O tolaryngol Head N eck Surg 1991;105:51-59.

25. Hazell JW P, Fraser J G, Robinson PJ. Positional audiometry in the diagnosis of perilymphatic fistula. A m J Otol 1992; 13(3):263-69.

26. Singleton GT. Diagnosis and treatment of perilymph fistulas without hearing loss. Otolaryngol Head Neck Surg 1986;94: 426-29.

27. Naiberg JB, Flemming E, Patterson M, Hawke $M$. The perilymphatic fistula: The end of an enigma? J Otolaryngol 1990; 19:260-63.

28. Hughes GB, Sismonis A, House JW. Is there consensus in perilymph fistula management? Otolaryngol Head Neck Surg 1990;102:111-17.

29. Daspit CP, Churchill D, Linthicum FH. Diagnosis of perilymph fistula using ENG and impedance. L aryngoscope 1980;90:217-23.

30. Fox EY, Balkany TJ , A renberg IK. The tuliophenomenon and perilymph fistula. Otolaryngol Head Neck Surg 1988;98:88-89.

31. Podoshin L, Fradis M, Ben-David J, Berger SI, Feiglin H. Perilymphatic fistula - the value of diagnostic tests. J L aryngol Otology 1994 July;108:560-63.

32. Gibson WPR. Electrocochleography in the diagnosis of perilymphatic fistula: Intraoperative observations and assessment ata new diagnostic official procedure. A mJ Otol 1992;13(2):146-51.

\section{ABOUT THE AUTHORS}

\section{Bachi T Hathiram}

Professor and Head, D epartment of ENT and Head and Neck Surgery Topiwala $\mathrm{N}$ ational M edical Collegeand BY L N air CharitableH ospital M umbai, M aharashtra, India

CorrespondenceAddres $\mathrm{Flat} N \mathrm{~N}$. 2, Ground F loor, M N B anaji B uilding Forjett Street Cross Lane, M umbai-400036, M aharashtra, India Phone: 91-9323699192, 91-9920499192, e-mail: bachi.hathiram@ rediffmail.com, orlclinics@gmail.com

\section{Vicky S Khattar}

A ssistant Professor, D epartment of ENT and H ead and N eck Surgery Topiwala $\mathrm{N}$ ational M edical Collegeand BY L N air Charitable H ospital M umbai, M aharashtra, India 